J. L. MORENO

F. FORRIOL

Laboratorio de Ortopedia Experimental. Departamento de Cirugía Ortopédica y Traumatología.

Clínica Universitaria

Facultad de Medicina.

Universidad de Navarra.

\section{Efecto de la congelación y del autoclave sobre las propiedades mecánicas del hueso cortical. Estudio experimental en fémur de cordero}

\author{
Effect of freezing and autoclave \\ processing on the mechanical \\ properties of cortical bone. \\ Experimental study in lamb femur
}

\section{Resumen}

Se ha estudiado la tensión máxima de rotura en aloinjertos congelados a $-20^{\circ} \mathrm{C}$ durante 60 días, tratados con autoclave, a $134^{\circ} \mathrm{C}$ y 8 minutos, y frescos, obtenidos de 54 fémures izquierdos de cordero. De cada uno de los fémures obtuvimos cilindros óseos de 5 $\mathrm{cm}$ de longitud que fueron cortados en tres porciones, caras anterior, externa e interna, que fueron ensayadas con el método de rotura a tres puntos.

Los resultados mostraron que el grupo congelado era más resistente $(p<0,05)$ que el grupo fresco y el tratado con autoclave ( $p$ $<0,05)$, mientras que no existían diferencias entre los dos últimos $(p<0,05)$. En la comparación de la tensión máxima de rotura a nivel de las caras, se apreciaba que la cara anterior era más resistente $(p<0,05)$, que la externa y la interna, sin diferencias significativas $(p>0,05)$ entre las dos uiltimas.

Los tratamientos de conservación afectan la tensión máxima que soporta el hueso con respecto al hueso fresco, provocando una mayor rigidez en los huesos congelados.

Palabras clave: Hueso. Tensión. Autoclave. Congelación. Aloinjerto.

\section{Summary}

A study was made of the maximum resistance to rupture of allografts frozen at $-20^{\circ} \mathrm{C}$ for 60 days, allografts processed $8 \mathrm{mi}$ nutes in an autoclave at $134^{\circ} \mathrm{C}$, and fresh allografts obtained from 54 left femurs from lambs. Five-centimeter-long bone cylinders were obtained from each femur and sectioned into three parts from the anterior, external, and internal faces. Allografts were assayed using the three-point rupture method.

The results showed that the frozen allografts were more resistant $(p<0.05)$ than either fresh or autoclave-processed allografts $(p<0.05)$. Comparison of the maximum resistance to rupture of the bone faces showed that the anterior face was more resistant ( $p$ $<0.05)$ than the external and internal faces, and the external and internal faces did not differ significantly $(p>0.05)$.

Preservation trealment modified maximum bone resistance in relation to fresh bone, with freezing increasing rigidity.

Key words: Bone. Resistance. Autoclave. Freezing. Allografts.

Correspondencia:

J. L. MORENO. Laboratorio de Ortopedia Experimental. Dpto. COT. CUN. Teléfono: (948) $425600 /(948) 296585.31080$ Pamplona. 


\section{Introducción}

Los injertos de hueso cortical son utilizados cada vez con mayor frecuencia en numerosas técnicas quirúrgicas tanto en la reconstrucción de defectos postraumáticos, como en deformidades congénitas y, especialmente, en la cirugía oncológica conservadora. En 1991, se trasplantaron 16.000 órganos en los Estados Unidos y se realizaron entre 150.000 y 300.000 trasplantes de hueso, cartílago, fascia, tendones y ligamentos ${ }^{12,26}$.

La conservación y el almacenado de hueso permite disponer de material de diferentes tamaños y en cantidad suficiente. Por otra parte, el método de conservación debe ser sencillo y evitar que el hueso pierda sus propiedades biológicas o mecánicas pues el injerto óseo cortical debe sustituir grandes defectos y restablecer la continuidad ósea siendo capaz de resistir y de transmitir solicitaciones esqueléticas.

El objetivo de nuestro trabajo es estudiar las características mecánicas de los aloinjertos y las variaciones que se originan al aplicar diferentes métodos de conservación (congelación y autoclave).

\section{Material y método}

Se utilizaron 54 fémures izquierdos, de cordero con edades comprendidas entre tres y cuatro meses. De cada uno de los fémures obtuvimos cilindros óseos de $5 \mathrm{~cm}$ de longitud, midiendo sus diámetros, interno y externo, con un calibre. Los cilindros óseos fueron cortados en tres porciones óseas correspondientes a las caras anterior, externa e interna (Fig. 1). En cada tira se midieron los grosores de la pared en tres secciones diferentes (central, proximal y distal), además del ancho y la altura de cada una de ellas. Cada una de estas tiras fue conservada según se especifica en la Tabla I. Diez muestras se rompieron durante el pretensado por lo que fueron excluidas del estudio.

Para conocer la tensión de rotura empleamos el método de rotura a tres puntos con una máquina de ensayos universal (Instron $4502^{\circledR}$ ) y con una mordaza homologada (Instron ${ }^{\circledR}$, $\mathrm{n}^{\mathrm{O}}$ ref. 2810-300). La velocidad de desplazamiento de la cruceta fue de $5 \mathrm{~mm} / \mathrm{min}$. Las condicromes ambiemtales fureron similares en todos los ensayos.

Conocida la carga máxima aplicada en el momento de la rotura, calculado el momento flector que produce y determinado el momento de inercia de la sección considerada se determinó la tensión máxima de rotura $(\sigma)$ que soporta el hueso en cada caso (Fig. 2).

Conocidos los datos geométricos de cada barra ósea se calculó el momento de inercia de la sección central para lo que aproximamos la sección del cilindro femoral a una sección regular ya que las variaciones obtenidas en el resultado por la aproximación empleada fueron despreciables como dedujimos calculando los momentos de inercia con otros datos medidos sobre los especímenes (Fig. 1).

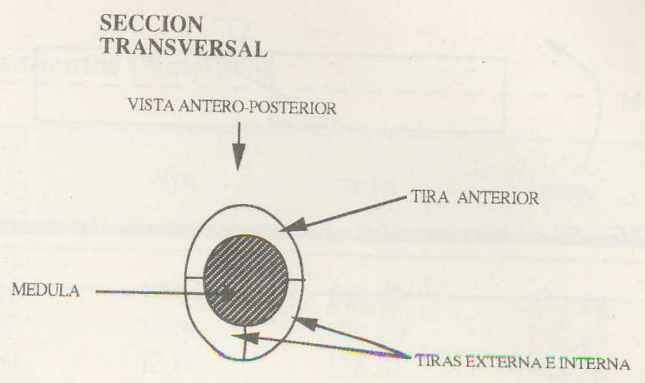

SECCION LONGITUDINAL
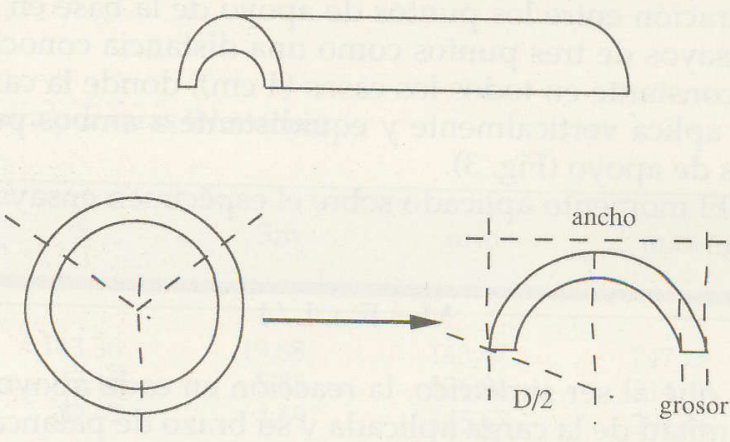

FIG. 1.-División del hueso en tres zonas para su ensayo y aproximación de la sección del cilindro femoral a una sección regular.

Tabla I. Distribución de las muestras en los grupos de ensayos a compresión

\begin{tabular}{|c|c|c|c|}
\hline Tratamiento & \multicolumn{2}{|c|}{ Cara } & Interna \\
\hline Fresco .......................................... & 21 & 21 & 20 \\
\hline Congelado a $-20^{\circ} \mathrm{C}$, 60 días ...... & 21 & 19 & 17 \\
\hline Autoclave a $134^{\circ} \mathrm{C}, 8$ minutos .. & 10 & 12 & 11 \\
\hline
\end{tabular}

La tensión se obtuvo con la fórmula:

$$
\sigma=\mathrm{My} / \mathrm{I}
$$

donde:

$\sigma=$ tensión

$\mathrm{M}=$ momento aplicado

$\mathrm{y}=$ distancia al centro de masa

$\mathrm{I}=$ momento de inercia de la sección

Para calcular el momento de inercia determinados, en primer lugar, la posición del centro de masas de la sección considerada. Luego, determinamos el momento de inercia respecto del centro geométrico de la 


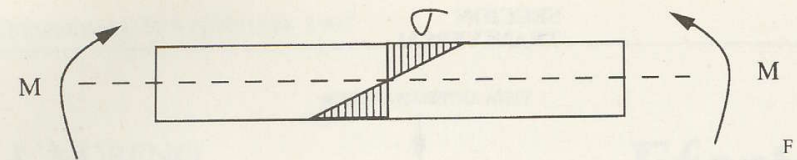

FIG. 2.-Distribución de tensiones en un esfuerzo a tensión.

sección completa del hueso sin cortar y aplicamos el teorema de los ejes paralelos de Steiner.

Para obtener el momento aplicado se definió la separación entre los puntos de apoyo de la base en los ensayos de tres puntos como una distancia conocida y constante en todos los casos $(4 \mathrm{~cm})$, donde la carga se aplica verticalmente y equidistante a ambos puntos de apoyo (Fig. 3).

El momento aplicado sobre el espécimen ensayado fue:

$$
\mathrm{M}=\mathrm{F} \times \mathrm{L} / 4
$$

ya que al ser simétrico, la reacción en cada apoyo es la mitad de la carga aplicada y su brazo de palanca la mitad de la distancia entre los puntos de apoyo.

Con este método pudimos determinar la tensión máxima soportada y donde se producía la rotura, en el lado sometido a tensión o en el de compresión, y comparar diferentes grupos de materiales ya que era un valor independiente de su geometría.

Se realizó el análisis estadístico de los valores haciendo referencia al tratamiento de conservación aplicado en cada muestra (congelado, autoclave y fresco) y la cara del fémur en la que se obtuvo (anterior, externa e interna). El paquete estadístico empleado fue el SPSS 4.0, para Macintosh.

Como paso previo el análisis estadístico, se comprobó que los valores obtenidos siguiesen una distribución normal mediante el test de Shapiro-Wilks,

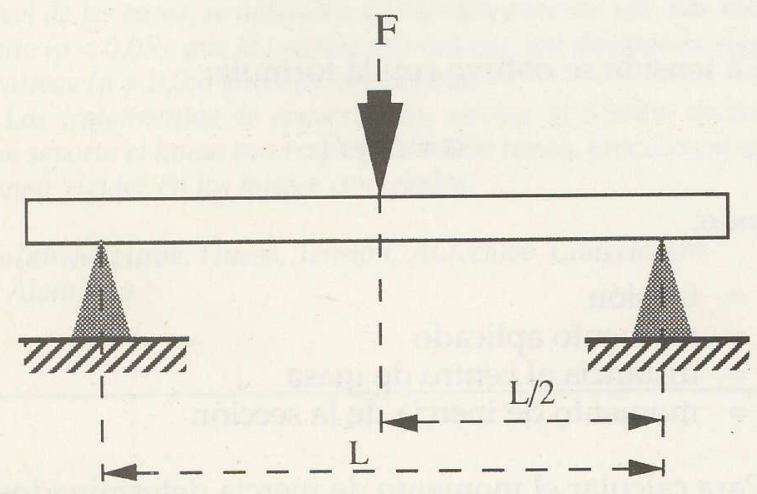

FIG. 3.-Esquema del ensayo a tres puntos. cumpliendo el requisito de homoscedasticidad, con el test de homogeneidad de varianzas de Levene. Tras esto se efectuó un análisis descriptivo (media aritmética, desviación estándar, error estándar, tamaño muestral, valor mínimo y máximo) y se compararon los valores de la tensión máxima de rotura entre los diferentes tratamientos y caras, mediante un análisis de la varianza de dos vías. En aquellos casos donde se detectaron diferencias estadísticamente significativas se efectuaron comparaciones múltiples post hoc mediante el test de Tukey.

\section{Resultados}

Con las mediciones de los parámetros morfométricos y la carga resistida por cada uno de los especímenes se efectuó el cálculo de la tensión máxima de rotura. Fue necesario aplicar a los datos una transformación matemática $(1 / x)$ para superar el requisito de normalidad y homoscedasticidad exigido en todas y cada una de las muestras.

La morfología de las diferentes porciones fueron homogéneas en todos los animales si bien las tres caras mostraron diferencias entre sí. La cara anterior presentaba una mayor convexidad; la cara interna era plana y regular, mientras que la cara externa presentaba una ligera torsión.

Los resultados de la Tabla II, III y IV, muestran que la tensión máxima de rotura en el grupo fresco de mayor valor corresponde a la cara anterior (368.41 $\mathrm{N})$, seguida por la externa $(196.11 \mathrm{~N})$ y la cara interna (181.81 N). En el grupo sometido a congelación, el comportamiento, es idéntico al del grupo fresco, el valor medio más alto corresponde a la cara anterior (450.72 N), el siguiente es el de la cara externa (254.53 N) y, por último, la cara interna (221.42 N). En el grupo sometido al autoclave, el orden cambia, el mayor valor de tensión máxima de rotura correspondía a, como en los casos anteriores, la cara anterior (318.77 N), seguida por la cara interna (197.99 N) y, por último, la externa (197.05 N), con valores muy semejantes.

Según el tratamiento aplicado y analizando los huesos en conjunto encontramos que el grupo que presentaba mayor tensión de rotura era el grupo congelado $(316.94 \mathrm{~N})$, después el grupo fresco $(240.86 \mathrm{~N})$ $\mathrm{y}$, por último, el grupo tratado con el autoclave $(234.25 \mathrm{~N})$

Una vez efectuada la descripción de los resultados obtenidos por tratamientos y zonas, se efectuó la comparación de los mismos por procedimientos estadísticos. En la comparación se confirma que los valores de tensión máxima de rotura en las diferentes caras del fémur no dependen de los tratamientos aplicados $(p=0,09)$ y aseguramos la ausencia de interacción entre los dos criterios implicados en el análisis.

Los valores de la tensión máxima de rotura se ven afectados por el proceso de conservación y almacena- 
Tabla II. Tensión máxima de rotura por tratamientos (Newtons)

\begin{tabular}{|c|c|c|c|c|c|c|}
\hline Tratamiento & Media & n & S & Sm & $\min$ & $\max$ \\
\hline 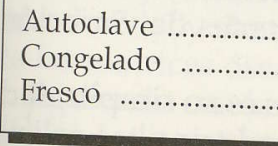 & $\begin{array}{l}234,25 \\
316,94 \\
249,86\end{array}$ & $\begin{array}{l}33 \\
57 \\
62\end{array}$ & $\begin{array}{r}98,44 \\
142,37 \\
107,60\end{array}$ & $\begin{array}{l}17,14 \\
18.86 \\
13,66\end{array}$ & $\begin{array}{l}143,47 \\
176.89 \\
136,81\end{array}$ & $\begin{array}{l}674,44 \\
747,25 \\
544,78\end{array}$ \\
\hline
\end{tabular}

min = valor mínimo $\quad \max =$ valor máximo $\quad S m=$ error estándar de la media

Tabla III. Tensión máxima de rotura por caras (Newtons)

\begin{tabular}{|c|c|c|c|c|c|c|}
\hline Caras & Media & n & S & $\mathrm{Sm}$ & $\min$ & $\max$ \\
\hline 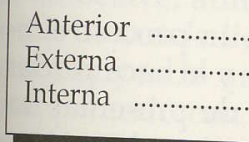 & $\begin{array}{l}392,11 \\
217,67 \\
199,55\end{array}$ & $\begin{array}{l}52 \\
52 \\
48\end{array}$ & $\begin{array}{r}143,36 \\
42,15 \\
32,47\end{array}$ & $\begin{array}{r}19,88 \\
5,85 \\
4,69\end{array}$ & $\begin{array}{l}143,47 \\
136,81 \\
145,63\end{array}$ & $\begin{array}{l}747,25 \\
314,48 \\
291,16\end{array}$ \\
\hline
\end{tabular}

Tabla IV. Tensión máxima de rotura por caras y tratamientos (Newtons)

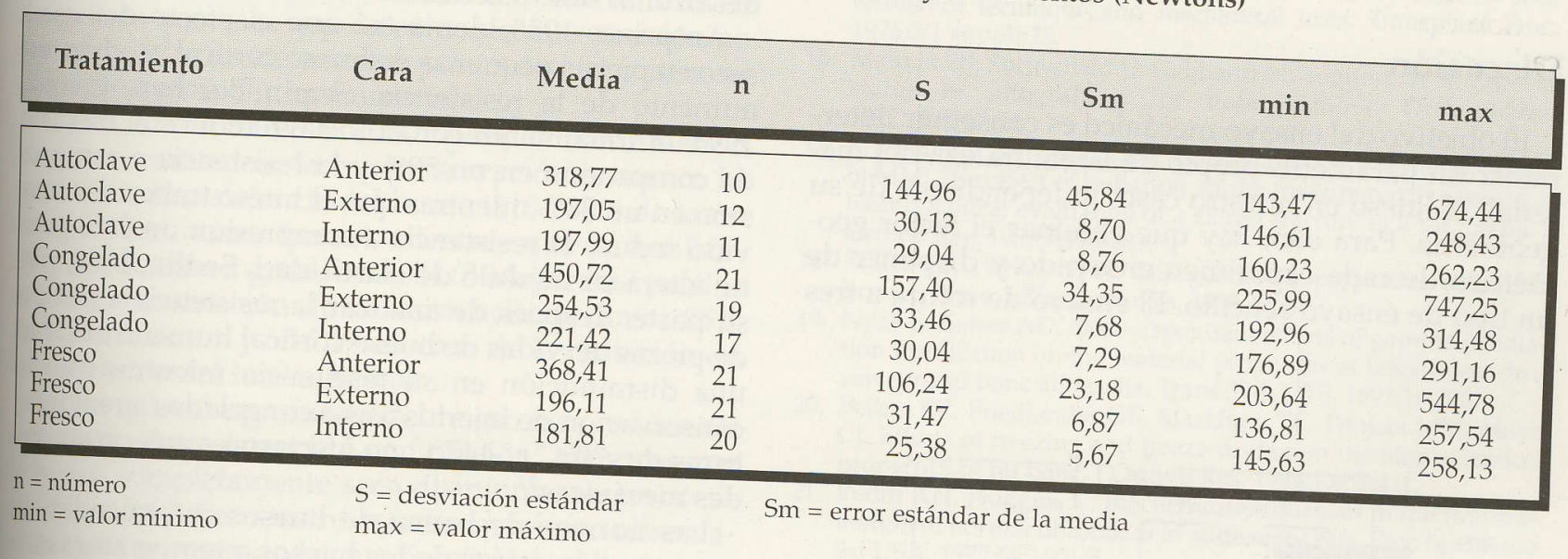

miento $(\mathrm{p}=1.0 \mathrm{E}-4)$, para detectar entre que tratamientos se establecieron las diferencias (Fig. 4) efectuando comparaciones múltiples pos hoc.

En las comparaciones entre los diferentes tratamientos detectamos la presencia de diferencias signigelación $(316.94)$ entre los huesos sometidos a conco (249.86 $)$, y los pertenecientes al grupo fresco $(249.86 \mathrm{~N})$, siendo más resistentes a la rotura los congelados. También detectamos diferencias significativas $(p<0,05)$ entre los huesos congelados (316.94 $\mathrm{N})$ y los sometidos al autoclave $(234.25 \mathrm{~N})$, siendo existen dentes los huesos congelados. Por último, no existen diferencias significativas ( $p>0,05)$, en los valores de tensión máxima de rotura, entre los huesos clave $(234.25 \mathrm{~N})$. y los sometidos al proceso de auto- 


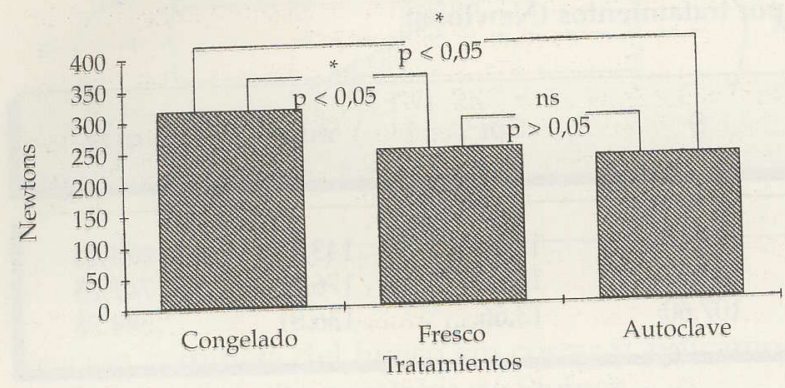

FIG. 4.-Comparación de los valores de tensión máxima de rotura entre los tratamientos.

En la comparación de la tensión máxima de rotura que soportan las tres caras del fémur estudiadas, se detectaron diferencias altamente significativas en dichos valores $(p=1.0 \mathrm{E}-4)$. Efectuamos comparaciones múltiples pos hoc, para detectar entre que caras se establecen las diferencias (Fig. 5), observando diferencias significativas $(p<0,05)$ entre la cara anterior $(392.11 \mathrm{~N})$ y la cara externa $(217.67 \mathrm{~N})$, siendo más resistente la anterior. También era más resistente la cara anterior $(392.11 \mathrm{~N})$ que la interna (199.55 $\mathrm{N}),(\mathrm{p}<0,05)$. En cambio, no se encontraron diferencias significativas $(p>0,05)$ en la tensión máxima de rotura, entre la cara externa $(217.67 \mathrm{~N})$ y la interna $(199.55 \mathrm{~N})$.

\section{Discusión}

El objetivo del ensayo mecánico es conseguir determinar un parámetro propio de la naturaleza del material, el hueso en nuestro caso, independiente de su geometría. Para ello, hay que eliminar el factor geométrico de cada espécimen ensayado y disponer de un tipo de ensayo sencillo. El ensayo de rotura a tres

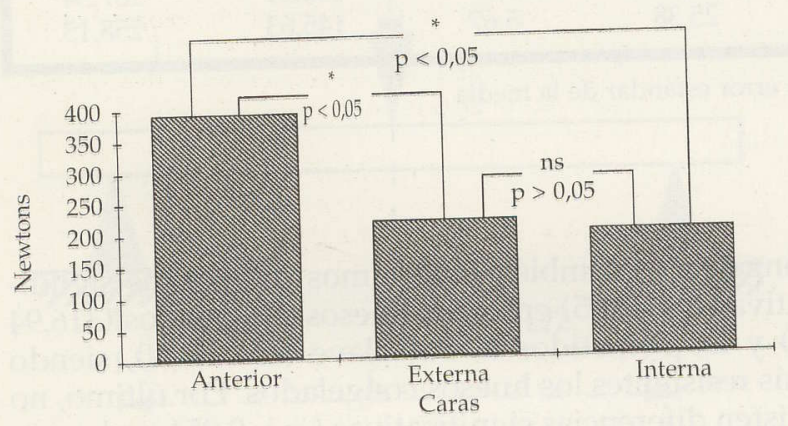

FIG. 5.-Comparación de los valores de tensión máxima de rotura entre las caras. puntos es un método sencillo que produce una solicitación a compresión en la cara superior y una solicitación a tensión en la cara inferior de la probeta, un método, al igual que el de los cuatro puntos, muy utilizado para el estudio de los materiales biológicos por presentar resultados reproducibles (coeficiente de variación entre 10 y 20\%) en comparación con los ensayos a torsión que presentan coeficientes de variación de hasta un $50 \% 5,6,29,30$

La ventaja de este tipo de ensayos es su simplicidad pero tienen el inconveniente de producir altas solicitaciones a cizallamiento cerca de la porción media del hueso que se puede solventar con ensayos a flexión a cuatro puntos aunque este tipo de ensayo precisa muestras exactamente iguales para conseguir las mismas fuerzas en cada punto de aplicación. Una condición importante es que la longitud del espécimen a ensayar sea lo suficientemente larga para garantizar la exactitud del ensayo, pues si fuese corta, muchos de los desplazamientos producidos por la carga serán producidos por solicitaciones a cizallamiento y no por solicitaciones a flexión.

El hueso tratado en el autoclave es un proceso simple que lo desvitaliza completamente y le hace perder sus propiedades mecánicas, además de presentar un alto poder antigénico ${ }^{11,13,14}$ por la desnaturalización y degradación de las proteínas, incluyendo las inductivas del hueso. El colágeno queda transformado por el calor en gelatina además de producirse una pérdida de agua importante a pesar de lo cual, según Taguchi y cols., 1995, el injerto tratado con el autoclave sirve como un elemento estructural sobre el cual se podrán desarrollar nuevas células.

Amprino, 1958, demostró que el efecto del calor seco en piezas pequeñas de hueso cortical produce un aumento de la resistencia. Según Borchers y cols., 1995, el tratamiento con autoclave reduce el módulo de compresión en un $59 \%$ y la resistencia a compresión en un $58 \%$ mientras que el hueso trabecular hervido reduce la resistencia a compresión un $26 \%$ pero no altera su módulo de elasticidad. Sedlin, 1966, por su parte, después de analizar la resistencia a flexión de piezas hervidas de hueso cortical humano observó una disminución en su resistencia mientras que la conservación de injertos óseos congelados a temperaturas de $-20^{\circ} \mathrm{C}$ a $-147^{\circ} \mathrm{C}$ no afectaron a sus propiedades mecánicas ${ }^{23}$.

Las normas de banco de huesos recomiendan la conservación estéril de los huesos a temperaturas inferiores a $-40^{\circ} \mathrm{C}$ para períodos superiores a los 6 meses o entre $-18^{\circ} \mathrm{C}$ y $-28^{\circ} \mathrm{C}$ para períodos inferiores a los 6 meses $^{8}$.

Por una parte, los procedimientos de congelación y liofilización no modifican las propiedades mecánicas del hueso trabecular mientras que la radiación y el autoclave de un injerto reducen las propiedades mecánicas y su capacidad de integración ${ }^{3,4,14}$. Altas dosis de radiación (> 3 Mrads) destruyen la mayor parte de las bacterias y virus en los tejidos humanos 
pero también reduce, aproximadamente en un 50\% la resistencia a compresión $4,15,21,27$. El nivel de radiadesconocida para inactivar el HIV en el hueso es que debe superio parece, por los estudios in vitro, que debe superar los 3 Mrads $^{19}$. Schwarz y cols., 1991, confirman los resultados presentados previamente por Urist y cols., 1976, señalando que dosis de radiación de 5-kGy destruyen la respuesta osteoinductiva de las matrices óseas desmineralizadas.

Siguiendo con la revisión de los resultados analizados en la literatura ${ }^{30}$, señalan que las radiaciones de rayos $\gamma(1 \mathrm{kGy}, 5 \mathrm{kGy})$ no disminuyen la fuerza de rotura del hueso cortical mientras que el tratado con autoclave a $134^{\circ}$ durante 5 minutos, disminuye la resistencia del hueso ( $28 \%$ del hueso control), aunque si se realiza durante 3 minutos, mantiene unas propieda-

cánicas entre el 1991, estudiaron las diferencias medistintas obstical conservado de formas distintas observando como su resistencia a la rotura en flexión, disminuye un $8,7 \%$ cuando se esteriliza en el autoclave, aumenta un 18,9\% con la liofilización y un $3,4 \%$ con la liofilización y la radiación $\gamma$ en argón, disminuyendo un 1,7\% cuando no se emplea este gas comparando con grupos controles de hueso congelado a $-25^{\circ}$ concluyendo que la resistencia a flexión no varía con la temperatura de conservación del hueso.

Por su parte, la liofilización disminuye la antigenicidad del injerto ya que es semejante a un proceso de desengrasado presentando una mejor incorporación que los aloinjertos congelados ${ }^{10}$ pero produce significantes reducciones en su resistencia a torsión y compresión $^{3,16,18,20,24,27}$, produciéndose microrroturas en el tejido lo que hace que este tipo de conservación no sea adecuada, desde nuestro punto de vista, para su utilización en la clínica humana.

Según Markbreiter y cols., 1989, y confirmando nuestros resultados, la criopreservación aumenta ligeramente la resistencia del hueso (110-120\% de la resistencia del hueso fresco) mientras que la liofilización o radiación a grandes dosis (3 Megarads) producen una disminución de la resistencia mecánica $55 \%$ la resistenciación y $65-70 \%$ en caso de la radiación de la resistencia del hueso fresco).

La congelación profunda del hueso no produce alteración en la resistencia a compresión ${ }^{7}$ mientras que el hueso completamente seco disminuye su resistencia en un $5 \% \%^{5}$. En nuestro estudio mecánico observamos, que las propiedades mecánicas del tejido óseo se ven afectadas por los procesos de conservación, siendo más resistentes los huesos congelados a $-20^{\circ} \mathrm{C}$, sin encontrar diferencias entre el grupo fresco y el trata-
do con el autoclave.

\section{Bibliografía} 1. Amprino R. Investigations on some physical properties of bone
tissue. Acta Anat. 1958:34:161-86.
2. Borchers RE, Gibson LJ, Burchardt H, Hayes WC. Effects of selected thermal variables on the mechanical properties of cular bone. Biomaterials. 1995;16:545-51.
Bright RW, Burstein AH properties of trabetical bone. Trans 24 th ORS $1978 ; 3$ properties of preserved cor-

4. Bright RW, Smarsch JD, Ga

bone by irradiation. En: Gambill VM. Sterilization of human (Eds). Osteochond En: Friedlander GE, Mankin HJ, Sell KW 1983:223-32

5. Currey JD. Mechanical properties of bone tissues with greatly differing functions. J Biomertes of bone tissues with greatly

6. Currey JD. The mechanical proper, 1979;12:313=9. 1970;73:210-31.

the mechanical properties. Effect of preservation medium on 1989;60:465-9.

8. Harrington

Limb salvage and prosthetic joufer HN, Luck JV, Moore TM. and selected high and replacement gy a sarcomas of bone after wide resection 1986;211:180-214

Heiple $\mathrm{KG}_{,} \mathrm{C}$

the healing process following $\mathrm{CH}$. A comparative study of plantation. I Bone Joint Surg different types of bone trans-

0. Jerosch I Muchow H, Clar maner Kortikalis nach versen $\mathrm{H}$. Uber die Stabilität von huSterilisations-verfach verschiedenen Konserviereungs- und

11. Johnston JO Harries T Orthop, 1991;129:295-301. salvage procedure for TJ, Alexander CE, Alexander AH. Limb tric total knee arthe knee by sphercengrafting. Clin Orthop 1983 and autogenous autoclaved bone

12. Justice KC, Recovery and ba181:137-45. rioper Nurs, 1993-2.90-7. banking; allograft bone. Seminars Pe-

1965:47(B):106-10.

toclaved Kreicbergs A, Strömberg L. Physical properties of au-

15. Komender J. Komen Orthop Scand. 1986;57:141=4. K. Radiation sterilized A, Dziedzic-Goclawska A, Ostrowski resonance techniqued bone grafts evaluated by electron spin 1976;8(1 suppl):25.

6. Mankin status of allografting, Gebhardt M, Tomford WW. Current 1992;15:1147-54.

bi MM: The LAfect Perkler RR, Friedländer GE, Peschel R, Panjabiomechanical evaluation on the fracture repair process: a Orthop Res. 1989:7:118-183. closed fracture in a rat model. J

18. Miller GJ Purkey WW. The

human tibiae. J BiomW. The geometric properties of paired

19. Nyak S Kaiser Biomechanics. 1980;13:1-8.

tion sterilization on . Dose-dependent effects of gamma irradiazen cortical bone allografts. Trans 37 th $O$ TS large fresch-fro-

20. Pelker RR, Friedlander GE, Trans 37th OTS. 1991;16:290.

CJ. Effects of freezing and Markham TC, Panjabi MM, Moen properties of rat bone and freeze-drying on the biomechanical properties of rat bone. J Orthop Res. 1984;1:405-411.

mation of normal fiochemical sequences in the transforSci USA, 1972:69:1601-5

22. Schwarz N, Schlag G, Thur

Redl H. Fresh autogen, Thurnher M, Eschberger J, Dinges HP geneic bone grafts in dogs. frozen allogeneic and decalcified allo-

23. Sedlin FD Hirsc physical properties of fetors affecting the determination of Scand 1966;37:29-48.

Chansky HA, Conrad EU, Chapman JR, Harrington RM screw pullout strenfth in hterilization and storage tratments on 1994:302:290-296.

25. Taguchi Y, Pereira BP, Kour AK, Pho RWH autograft bone combined with vascularized bs. Autoclaved marrow. Clin Orthop. 1995;320:220-30 
26. Tomford WW. Transmission of disease through transplantation of musculoskeletal allografts. J Bone Joint Surg. 1995;77A: $1742-54$

27. Triantafyllou N, Sotiorpoulus E, Triantafyllou J. The mechanical properties of lyophylized and irradiated bone grafts. Acta Orthop Bel. 1975;41:35-41.

28. Urist MR. Practical applications of basic research on bone graft physiology. AAOS. 1976;25:1-26
29. Voggenreiter G, Ascherl R, Blümel G, Schmit-Neuerburg KP. Effects of preservation and sterilization on cortical bone grafts A scanning electron microscopic study. Arch Orthop Trauma Surg. 1994;113:294-6.

30. Voggenreiter G, Ascherl R, Früh HJ, Blümel G, Schmit-Neuerburg KP. Konservierung und sterilisationvon Kortikalis-biomechanische Untersuchungen an der Ratte. Unfallchirurg. 1995; $98: 53-8$.

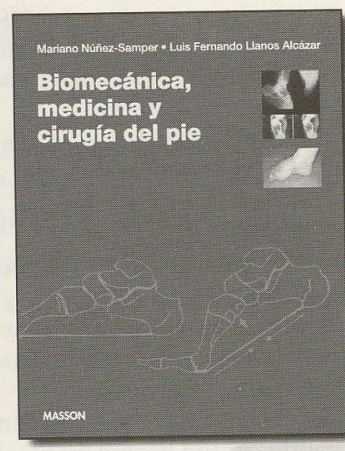

\title{
Biomecánica,
} medicina y cirugía del pie

\author{
M. Núñez-Samper y L.F. Llanos Alcázar
}

El pie PASO a PASO

Una visión completa, actualizada y armónica de una de las patologías más frecuentes de la clínica cotidiana en traumatología y patología del aparato locomotor.

- Los temas que han recibido especial atención son:

- Biomecánica: con material de investigación reciente.

- Exploración: nuevas técnicas.
- Patología congénita y adquirida: enfoques actuales.

- Técnicas quirúrgicas.

INDICE DE CAPITULOS (extracto): PARTE I. Ciencias Básicas. Sección A. Evolución y desarrollo del pie I. Concepto histórico del pie 2. Fi logenia del pie 3. Desarrollo del tobillo y el pie. Sección B. Anatomía del pie 4. El pie óseo 5 . Miología, vascularización e inervación del pie 6. Anatologenia del pie 3. Desaŕón C. Biomecánica 7. Biomorfología 8. Cinemática y cinética 9. Estructura funcional del pie 10. Marcha humana. Sección D. Exmía de superficie. Sección C. Biomecanica . Bación complementaria específica del pie 13. Exploración del movimiento y de la marcha 14 . Diagnóstico ploracion I. Exploracion clinica I2. Exploracion compléficas del pie. PARTE II. Pie infantil y puberal. Sección A. Pie en crecimiento I6. Cronolode imagen 15. Exploraciones complementarias inespecifcas del pie. PÁ B. Trostornos del desarrollo 19. Defectos congénitos 20. Coaliciones tarsianas gía del pie 17. Radiología del pie infantil 18. Osteocondrosis. Seccion B. Sección C. Deformidades adquiridas 23. Pie plano 24. Pie cavo. PARTE III. 21. Pie equinovaro 22. Alteraciones del tarso posterior y metatarsianos. Seccion C. Deformidades adquiridas 23 . Pección A. Deformidades adquiridas del antepie 25. Sindrome de insuficiencia del primer radio 26 . Sindrome de sobrecarga del primer radio 27. Sindrome de sobrecarga anterior y sindrome de insuficiencia de los racio medios 20. Deformidades de los dedos 29. Metatarsalgias de origen no mecánico. Sección B. Patologia adquirida 30. Tumores del pie 31. Pie vascular y pie diabetico 32. Pie reumático 33. Inecciones oseas 34. Lesiones deportivas. El pie en la danza. PARTE IV. Pie neurologico. 35. Parálisis flaccidas del pie 36. Deformidaces en la paralisis cerebral espastica 37. El pie en la espina bifica 38. El pie en las neuropatias periféricas 39. Pie neuropatico. PARTE V. Patologia de partes blandas y lesiones de la piel. 40. Talalgias 41 . Síndrome del seno del tarso y síndromes canaliculares 42. Lesiones de la piel y patologia ungueal 43. Quemaduras y de la pies congelacione de tobillo 46. Fracturas del pilón tibial 47. Facturas de los huesos del tarso 48. Luxaciones periastragalinas 49. Fracturas y luxaturas-luxain ciones de los metatas an rugia reparadora de las peras rurgicas. 56. Vas de acceso. An sis y osteotaxis 59 . Osteotomias 60 . Ar putaciones y desarticulaciones. PArétes. El calzado 68. Manifestaciones de las enfermedades sistémicas en el pie. Indice alfabético de materias.

$\square$ Sí, deseo remitan a mi nombre estad

NÚÑEZ-SAMPER: BIOMECÁNICA MEDICINA Y CIRUGÍA DEL PIE

PVP: 17.500 Ptas. con IVA y 16.827s

FORMA DE PAGO:

Contra reembolso sin cargo alguno.

Nombre

$1^{\text {er Apellido }}$

$2^{\circ}$ Apellido

Año nacimiento

Teléfono

Especialidad

Dirección

Localidad

FIRMA

Puede dirigirse a su librería habitual o remitir esta Teretale

MASSON, S.A.

Ronda General Mitre, 149

08022 BARCELONA (España)

Fax: 93-253 0515

e-mail: grupo masson@bcn.servicom.es

10710 9 